Journal of Engineering and Applied Sciences 15 (6): 1487-1495, 2020

ISSN: 1816-949X

(C) Medwell Journals, 2020

\title{
Fixed Point Results on Generalized Multiplicative Contraction Mappings in Extended b-Multiplicative Metric Spaces
}

\author{
Budi Nurwahyu \\ Department of Mathematics, Hasanuddin University, Campus Tamalanrea KM 10, \\ 90245 Makassar, Indonesia
}

\begin{abstract}
This study introduces the notion of extended multiplicative b-metric space and proves the existence and uniqueness of fixed point for generalized multiplicative contraction mappings in the extended multiplicative b-metric space. Some examples in main results are provided.
\end{abstract}

Key words: Fixed point, weakly compatible function, multiplicative contraction, extended multiplicative b-metric

\section{INTRODUCTION}

Fixed point is one of the important thing in mathematical analysis and most useful to determine an existence of solution differential equation system, integral equation, roots of function, etc.

Bashirov et al. (2008) introduced a notion of multiplicative metric space. Some authors have contributed to the use of the multiplicative metric space for fixed point results such as by Abbas et al. (2015) and Abdou (2016). Similarly, Khan et al. (2017) used the space for fixed point results on integral-type inequality and Singh et al. (2016) proposed and proved fixed point theorems on expansive mapping. However, some researchers utilized the space for common fixed point results on multiplicative contraction such as He et al. (2014) who proved common fixed point theorems on weak commutative mapping and $\mathrm{Gu}$ and Cho (2015) who proved common fixed point theorems of four maps on multiplicative contraction mapping. Kumar et al. (2016) proposed and proved random fixed point theorems on multiplicative contraction mapping. Abdou (2016) and Khan and Imdad (2016) used compatible and coincidence property for common fixed point on generalized multiplicative contraction mappings. In 2017, Ali et al. (2017) introduced b-multiplicative metric space as a generalization of multiplicative metric space for fixed point results on multiplicative contraction mapping with an application in Fredholm integral equation.

In this study, we introduce a new notion of an extended b-multiplicative metric space as a generalization of b-multiplicative metric space and prove some fixed point and common fixed point theorems on generalized multiplicative contraction mapping. Moreover, we also provide some examples to show the clarification of the theorems.

\section{MATERIALS AND METHODS}

Preliminaries: In this study, we show some definitions and examples that will be used in the main results.

Definition 2.1; Bashirov (2008): Let, $X$ be a nonempty set. A mapping d: $X \times X \rightarrow[1, \infty)$ is said to be multiplicative metric, if $\mathrm{d}$ satisfies the following conditions:

- $\quad d(x, y)=1$ if and only if $x=y$

- $\quad d(x, y)=d(y, x)$

- $\quad d(x, y) \leq d(x, z) \cdot d(z, y)$, for all $x, y, z \in X$

If $\mathrm{d}$ is a multiplicative metric on $\mathrm{X}$, the pair $(\mathrm{X}, \mathrm{d})$ is called a multiplicative metric space.

Remark 2.1: If $(X, d)$ is a metric space then $(X, \rho)$ is a multiplicative space where $\rho(x, y)=a^{d(x, y)}$ with $a>1$.

Remark 2.2: If $(X, d)$ is a multiplicative metric space then $1 / d(x, y) \leq d(x, z) / d(z, y) \leq d(x, y)$.

Example 2.1; Bashirov (2008): Let $X=(0, \infty)$, define $\mathrm{d}(\mathrm{x}, \mathrm{y})=|\mathrm{x} / \mathrm{y}|^{*}$ where $|.|^{*}: \mathrm{X} \rightarrow \mathrm{X}$ by:

$$
|x|^{*}=\left\{\begin{array}{l}
x, \text { if } x \geq 1 \\
\frac{1}{x}, \text { if } x<1
\end{array}\right.
$$

Then $\mathrm{d}$ is a multiplicative metric.

Example 2.2; He (2014): Let $X=R$, define $d(x, y)=a^{|x-y|}$ where $(X, \rho)$ is a metric space and $a>1$. Then, $d$ is a multiplicative metric.

Example 2.3; He (2014): Let $X=R$, define $d(x, y)=$ $a^{\rho(x, y)}$ where $(X, \rho)$ is a metric space and $a>1$. Then $d$ is a multiplicative metric. 
Remark 2.3: Multiplicative metric space and metric space are independent each other. As shown in Example 2.1, we have:

$$
\mathrm{d}\left(\frac{1}{2}, 1\right)+\mathrm{d}\left(\frac{3}{2}, 2\right)=2+\frac{4}{3}=\frac{10}{3}<4=\mathrm{d}\left(\frac{1}{2}, 2\right)
$$

The example shows that multiplicative metric does not necessary be metric as it does not satisfy triangular inequality in metric. On the other hand, the usual metric on $\mathrm{R}$ does not necessary be multiplicative metric, since:

$$
\rho\left(1, \frac{1}{2}\right) \cdot \rho\left(2, \frac{3}{2}\right)=\left|1-\frac{1}{2}\right|\left|2-\frac{3}{2}\right|=\frac{1}{4}<\frac{1}{2}=\left|1-\frac{3}{2}\right|=\rho\left(1, \frac{3}{2}\right)
$$

Definition 2.2; Ali et al. (2017): Let $X$ be a nonempty set and let $b \geq 1$. A mapping $d_{b}: X \times X \rightarrow[1, \infty)$ is said to be b-multiplicative metric, if $\mathrm{d}_{\mathrm{b}}$ satisfies the following conditions:

$$
\begin{aligned}
& d_{b}(x, y) \geq 1 \text { and } d_{b}(x, y)=1 \text { if and only if } x=y \\
& d_{b}(x, y)=d_{b}(x, y) \\
& d_{b}(x, y) \leq\left(d_{b}(x, z)\right)^{b} \cdot\left(d_{b}(z, y)\right)^{b} \text { for all } x, y, z \in X
\end{aligned}
$$

If $d$ is a multiplicative metric on $X$, the pair $(X, d)$ is called a b-multiplicative metric space.

Example 2.4: Let $X=R$, define $d(x, y)=a^{|x-y| p}$ where $x$, $\mathrm{y} \in \mathrm{X}$ and $\mathrm{a}>1, \mathrm{p}>1$. Then $\mathrm{d}$ is b-multiplicative metric with $\mathrm{b}=2^{\mathrm{p}-1}$.

Example 2.5; He (2014): Let $X=R$, define $d(x, y)=a^{(\rho(x, y))^{p}}$ where $(X, \rho)$ is a metric space and $a>1$, $\mathrm{p}>1$. Then $\mathrm{d}$ is a b-multiplicative metric with $\mathrm{b}=2^{\mathrm{p}-1}$.

Definition 2.3: Let $X$ be a nonempty set and b: $X \times X \rightarrow[1, \infty)$ be a mapping. A mapping $d_{b}: X \times X \rightarrow[1, \infty)$ is said to be an extended b-multiplicative metric, if $d_{b}$ satisfies the following conditions:

$$
\begin{aligned}
& d_{b}(x, y)=1 \text { if and only if } x=y \\
& d_{b}(x, y)=d_{b}(y, x) \\
& d_{b}(x, y)\left(d_{b}(x, z)\right)^{b(x, y)} \cdot\left(d_{b}(z, y)\right)^{b(x, y)} \text { for all } x, y, z X
\end{aligned}
$$

If $\mathrm{d}$ is an extended multiplicative metric on $\mathrm{X}$, then the pair $(\mathrm{X}, \mathrm{d})$ is called an extended b-multiplicative metric space

Example 2.6: Let $X=[0,1]$, define $b(x, y)=2+|x-y|^{2}$ and $d_{b}(x, y)=a^{|x-y| 2}$ where $x, y \in X$ and $a>1$. Then $d$ is an extended b-multiplicative metric. All conditions are obvious for $\mathrm{x}=\mathrm{y}$. For $\mathrm{x} \neq \mathrm{y}$ is also obvious for first and second condition. For the third condition, consider that:

$$
\begin{aligned}
& d_{b}(x, y)=a^{|x-y|^{2}} \leq a^{2\left((x-z)^{2}+(z-y)^{2}\right)} \leq a^{\left(2+|x-y|^{2}\right)\left((x-z)^{2}+(z-y)^{2}\right)}= \\
& \mathrm{a}^{\mathrm{b}(\mathrm{x}, \mathrm{y})\left((\mathrm{x}-\mathrm{z})^{2}+(\mathrm{z}-\mathrm{y})^{2}\right)}=\mathrm{a}^{\mathrm{b}(\mathrm{x}, \mathrm{y})\left((\mathrm{x}-\mathrm{z})^{2}+(\mathrm{z}-\mathrm{y})^{2}\right)}= \\
& a^{b(x, y)(x-z)^{2}} a^{b(x, y)(z-y)^{2}}=\left(d_{b}(x, z)\right)^{b(x, y)} \cdot\left(d_{b}(z, y)\right)^{b(x, y)}
\end{aligned}
$$

Hence, $d_{b}$ is an extended b-multiplicative metric.

Definition 2.4: Let $\left(X, d_{b}\right)$ be an extended b-multiplicative space and let $\left\{a_{n}\right\}$ be a sequence in $X$.

- $\left\{a_{n}\right\}$ is said to be a convergent to $a \in X$ if for every $\varepsilon>1$ there exists $K \in N$ such that $n \geq K$, then $d_{b}\left(a_{n}, a\right)<\varepsilon$ that is $d_{b}\left(a_{n}, a\right) \rightarrow 1$ as $n \rightarrow \infty$

- $\quad\left\{a_{n}\right\}$ is said to be a Cauchy if for every for every $\varepsilon>1$ there exists $K \in N$ such that $n, m \geq K$, then $d_{b}\left(a_{n}, a_{m}\right)<\varepsilon$ that is $d_{b}\left(a_{n}, a_{m}\right) \rightarrow 1$ as $n, m \rightarrow \infty$

- $\quad \mathrm{X}$ is called a complete, if every Cauchy sequence in an extended b-multiplicative space $\left(X, d_{b}\right)$ is convergent in $\mathrm{X}$

Remark 2.4: In an extended b-multiplicative space, a convergent sequence is convergent to a unique limit point. It is shown in this lemma as follows:

Lemma 2.1: Let $\left(X, d_{b}\right)$ be an extended multiplicative b-metric space and $\left\{x_{n}\right\}$ be a convergent sequence in $X$. Then $\left\{x_{n}\right\}$ is convergent to a unique limit point.

Proof: Suppose that $d_{b}\left(x_{n}, x\right) \rightarrow 1$ and $d_{b}\left(x_{n}, y\right) \rightarrow 1$ as $n \rightarrow \infty$ by using multiplicative triangular inequality, we obtain:

$$
d_{b}(x, y) \leq\left(d_{b}\left(x, x_{n}\right)\right)^{b(x, y)}\left(d_{b}\left(x_{n}, y\right)\right)^{b(x, y)} \rightarrow 1 \text { as } n \rightarrow \infty
$$

Hence by using condition 1 in $(1)$, we have $d_{b}(x, y)=$ 1 that is $\mathrm{x}=\mathrm{y}$.

Definition 2.5; He (2014): Let (X, d) be a multiplicative space. A mapping $\mathrm{f}: \mathrm{X} \rightarrow \mathrm{X}$ is said to be a multiplicative contraction, if there exists $\lambda \in(0,1)$ such that satisfies the following conditions: $d(f x, f y) \leq d^{\lambda}(x, y)$, for all $x, y \in X$.

$$
d(f x, f y) \leq d^{\lambda}(x, y)
$$

Definition 2.6; Khan and Imdad (2016): Let $X$ be a nonempty set and $T_{1}, T_{2}: X \rightarrow X$ be self-mappings. $\left\{T_{1}, T_{2}\right\}$ is called weakly compatible for any $\mathrm{x} \in \mathrm{X}$, if $\mathrm{T}_{1} \mathrm{x}=\mathrm{T}_{2} \mathrm{x}$ then $\mathrm{T}_{2} \mathrm{~T}_{1} \mathrm{x}=\mathrm{T}_{1} \mathrm{~T}_{2} \mathrm{x}$.

\section{RESULTS AND DISCUSSION}

In the beginning of this section and by using multiplicative contraction for complete extended multiplicative b-metric space, we establish and prove some fixed point theorems. Moreover, we prove a fixed point and common fixed point on a contraction-type mapping. 
Theorem 3.1: Let $\left(X, d_{b}\right)$ be a complete extended multiplicative b-metric space and $\mathrm{f}: \mathrm{X} \rightarrow \mathrm{X}$ be a mapping that satisfies the following conditions:

$$
b(x, y) d_{b}(f x, f y) \leq d_{b}^{\lambda}(x, y)
$$

where $0<\lambda<1$. If:

$$
\lim _{n, m \rightarrow \infty} b\left(x_{n}, x_{m}\right)<\frac{1}{\lambda}
$$

then $\mathrm{f}$ has a fixed point.

Proof: Let taking $\mathrm{x}_{\mathrm{o}} \in \mathrm{X}$ and let $\left\{\mathrm{x}_{\mathrm{n}}\right\}$ be a sequence where $\mathrm{x}_{\mathrm{n}}=\mathrm{f}\left(\mathrm{x}_{\mathrm{n}-1}\right)$ for $\mathrm{n}=1,2,3, \ldots$ By using Eq. 2, we have:

$$
b\left(x_{n-1}, x_{n}\right) d_{b}\left(x_{n}, x_{n+1}\right)=b\left(x_{n-1}, x_{n}\right) d_{b}\left(f x_{n-1}, f x_{n}\right) \leq d_{b}^{\lambda}\left(x_{n-1}, x_{n}\right)
$$

Thus, we get:

$$
d_{b}\left(x_{n}, x_{n+1}\right) \leq \frac{d_{b}^{\lambda}\left(x_{n-1}, x_{n}\right)}{b\left(x_{n-1}, x_{n}\right)} \leq d_{b}^{\lambda}\left(x_{n-1}, x_{n}\right)
$$

Recursively, we have:

$$
\mathrm{d}_{\mathrm{b}}\left(\mathrm{x}_{\mathrm{n}}, \mathrm{x}_{\mathrm{n}+1}\right) \leq \mathrm{d}_{\mathrm{b}}^{\lambda^{\mathrm{n}}}\left(\mathrm{x}_{0}, \mathrm{x}_{1}\right)
$$

Since, $0<\lambda<1$ and using Eq. 3, we have $d_{b}\left(x_{n}, x_{n+1}\right) \rightarrow 1$ as $n \rightarrow \infty$. Next, we have to show that $\left\{x_{n}\right\}$ is a Cauchy sequence. Consider that for all $n, m \in N$ with $m>n$ by the multiplicative triangle inequality in Eq. 1 and using Eq. 3, we have:

$$
\begin{aligned}
& d_{b}\left(x_{n}, x_{m}\right) \leq\left(d_{b}\left(x_{n}, x_{n+1}\right)\right)^{b\left(x_{n}, x_{m}\right)} \times\left(d_{b}\left(x_{n+1}, x_{m}\right)\right)^{b\left(x_{n}, x_{m}\right)} \leq \\
& \left(d_{b}\left(x_{0}, x_{1}\right)\right)^{\lambda^{n} b\left(x_{n}, x_{m}\right)} \times\left(d_{b}\left(x_{n+1}, x_{n+2}\right)\right)^{b\left(x_{n}, x_{m}\right) b\left(x_{n+1}, x_{m}\right)} \times \\
& \left(d_{b}\left(x_{n+2}, x_{m}\right)\right)^{b\left(\left(x_{n}, x_{m}\right) b\left(x_{n+1}, x_{m}\right)\right.} \leq\left(d_{b}\left(x_{0}, x_{1}\right)\right)^{\lambda^{n} b\left(x_{n}, x_{m}\right)} \times \\
& \left(d_{b}\left(x_{0}, x_{1}\right)\right)^{\lambda^{n+1} b\left(x_{n}, x_{m}\right) b\left(\left(x_{n+1}, x_{m}\right)\right.} \times\left(d_{b}\left(x_{n+2}, x_{m}\right)\right)^{b\left(x_{n}, x_{m}\right) b\left(x_{n+1}, x_{m}\right)} \leq(4) \\
& \left(d_{b}\left(x_{0}, x_{1}\right)\right)^{\lambda^{n} b\left(x_{n}, x_{m}\right)} \times\left(d_{b}\left(x_{0}, x_{1}\right)\right)^{\lambda^{n+1} b\left(x_{n}, x_{m}\right) b\left(x_{n+1}, x_{m}\right)} \times \\
& \left(d_{b}\left(x_{0}, x_{1}\right)\right)^{\lambda^{m-1} b\left(x_{n}, x_{m}\right) b\left(x_{m-1}, x_{m}\right)} \\
& \left(d_{b}\left(x_{0}, x_{1}\right)\right)^{\sum_{j=0}^{m-n-1} \lambda^{n+j} \prod_{i=0}^{j} b\left(x_{n+1}, x_{m}\right)}
\end{aligned}
$$

In fact, since $\mathrm{b}\left(\mathrm{x}_{\mathrm{i}}, \mathrm{x}_{\mathrm{m}}\right) \geq 1$ by using Eq. 4 we:

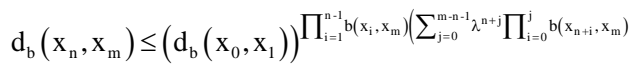

Let:

$$
\mathrm{S}_{\mathrm{n}}=\sum_{\mathrm{j}=1}^{\mathrm{n}} \lambda^{\mathrm{j}} \prod_{\mathrm{i}=1}^{\mathrm{j}} \mathrm{b}\left(\mathrm{x}_{\mathrm{i}}, \mathrm{x}_{\mathrm{m}}\right)
$$

Then by using Eq. 5 we obtain:

$$
\mathrm{d}_{\mathrm{b}}\left(\mathrm{x}_{\mathrm{n}}, \mathrm{x}_{\mathrm{m}}\right) \leq\left(\mathrm{d}_{\mathrm{b}}\left(\mathrm{x}_{0}, \mathrm{x}_{1}\right)\right)^{\mathrm{S}_{\mathrm{m}-1-\mathrm{S}_{\mathrm{n}-1}}}
$$

Let:

$$
a_{j}=\lambda^{j} \prod_{i=0}^{j} b\left(x_{i}, x_{m}\right)
$$

We have:

$$
\mathrm{S}_{\mathrm{n}}=\sum_{\mathrm{j}=1}^{\mathrm{n}} \mathrm{a}_{\mathrm{j}}
$$

By using the ratio test and since, $\lim _{n, m \rightarrow \infty} b\left(x_{n}, x_{m}\right)<\frac{1}{\lambda}$, we have $\operatorname{Lim}_{j \rightarrow \infty} \frac{a j}{a_{j-1}}=\lim _{j \rightarrow \infty} \lambda b\left(x_{j}, x_{m}\right)=\lambda \lim _{j \rightarrow \infty} b\left(x_{j}, x_{m}\right)<1$ for each $m \in N$. Thus, we have $\left\{S_{n}\right\}$ is convergent for each $m \in N$, this implies $\lim _{\mathrm{n}, \mathrm{m} \rightarrow \infty} \mathrm{S}_{\mathrm{m}-1}-\mathrm{S}_{\mathrm{n}-1}=0$. Then by using Eq. 6, we get $d_{b}\left(x_{n}, x_{m}\right) \rightarrow 1$ as $n, m \rightarrow \infty$. Hence, $\left\{x_{n}\right\}$ is a Cauchy sequence. Since, $\mathrm{X}$ is complete, there exists $\mathrm{X}^{*} \in \mathrm{X}$ such that $d_{b}\left(x_{n}, x^{*}\right) \rightarrow 1$ as $n \rightarrow \infty$. Claim that is the fixed point of $f$. Consider that:

$$
\begin{aligned}
& d_{b}\left(f x^{*}, x^{*}\right) \leq\left(d_{b}\left(f x^{*}, x_{n+1}\right)\right)^{b\left(f x^{*}, x^{*}\right)}\left(d_{b}\left(x_{n+1}, x^{*}\right)\right)^{b\left(f x^{*}, x^{*}\right)}= \\
& \left(d_{b}\left(f x^{*}, f x_{n}\right)\right)^{b\left(f x^{*}, x^{*}\right)}\left(d_{b}\left(x_{n+1}, x^{*}\right)\right)^{b\left(f x^{*}, x^{*}\right)} \leq \\
& \left(\frac{1}{b\left(x^{*}, x_{n}\right)}\right)^{b\left(f x^{*}, x^{*}\right)}\left(d_{b}\left(x^{*}, x_{n}\right)\right)^{\lambda b\left(f x^{*}, x^{*}\right)} \times\left(d_{b}\left(x_{n+1}, x^{*}\right)\right)^{b\left(f x^{*}, x^{*}\right)} \leq \\
& \left(d_{b}\left(x^{*}, x_{n}\right)\right)^{\lambda b\left(f x^{*}, x^{*}\right)}\left(d_{b}\left(x_{n+1}, x^{*}\right)\right)^{b\left(f x^{*}, x^{*}\right)}
\end{aligned}
$$

Since, $d_{b}\left(x_{n}, x^{*}\right) \rightarrow 1$ as $n \rightarrow \infty$ then from Eq. 7 , we get $d_{b}\left(f x^{*}, x^{*}\right)=1$ that is $f x^{*}=x^{*}$. Hence, $x^{*}$ is the fixed point of $f$. For uniqueness of the fixed point, suppose $u^{*}$ is another fixed point of $\mathrm{f}$ that is $\mathrm{fu}^{*}=\mathrm{u}^{*}$. Consider that $d_{b}\left(u^{*}, x^{*}\right)=d_{b}\left(f u^{*}, f x^{*}\right) \leq 1 / b\left(u^{*}, x^{*}\right)\left(d_{b}\left(u^{*}, x^{*}\right)\right)^{\lambda} \leq\left(d_{b}\left(u^{*}, x^{*}\right)\right)^{\lambda}$. Thus we get $\left(d_{b}\left(u^{*}, x^{*}\right)\right)^{1-\lambda} \leq 1$. Since, $1-\lambda>0$ and $\left(d_{b}\left(u^{*}\right.\right.$, $\left.\left.x^{*}\right)\right) \geq 1$, we get $d_{b}\left(u^{*}, x^{*}\right)=1$ that is $u^{*}=x^{*}$.

Example 3.1: As shown in Example 2.4 and by taking $\mathrm{a}=4^{32}$ that is $\mathrm{b}(\mathrm{x}, \mathrm{y})=2+(\mathrm{x}-\mathrm{y})^{2}$ and $\mathrm{d}_{\mathrm{b}}(\mathrm{x}, \mathrm{y})=$ $(4)^{32|x-y| 2}$ where $x, y \in X=[0,1]$. Let $f(x)=x / 4$, for $x, y \in X$. Let $\mathrm{x} \neq \mathrm{y}$, consider that:

$$
\begin{aligned}
& d_{b}(f x, f x)=d_{b}\left(\frac{x}{4}, \frac{y}{4}\right) \leq(4)^{32\left|\frac{x}{4}-\frac{y}{4}\right|^{2}}=(4)^{\frac{32}{8}|x-y|^{2}-\frac{32}{16}|x-y|^{2}}= \\
& \frac{(4)^{\frac{32}{8}|x-y|^{2}}}{(4)^{\frac{32}{16}|x-y|^{2}}}=\frac{(4)^{\frac{32}{8}|x-y|^{2}}}{(4)^{2|x-y|^{2}}} \leq \frac{(4)^{\frac{32}{8}|x-y|^{2}}}{2 e^{|x-y|^{2}}} \leq \frac{(4)^{\frac{32}{8}|x-y|^{2}}}{2\left(1+|(x-y)|^{2}\right)} \leq \\
& \frac{(4)^{\frac{32}{8}|x-y|^{2}}}{2+|(x-y)|^{2}} \leq \frac{\left((4)^{32|x-y|^{2}}\right)^{\frac{1}{8}}}{2+|(x-y)|^{2}}=\frac{\left(d_{b}(x, y)\right)^{\frac{1}{8}}}{b(x, y)}=\frac{\left(d_{b}(x, y)\right)^{\lambda}}{b(x, y)}
\end{aligned}
$$

Hence, we obtain:

$$
b(x, y) d_{b}(f x, f y) \leq\left(d_{b}(x, y)\right)^{\lambda}
$$


where $\lambda=1 / 8<1$. By using $\mathrm{f}(\mathrm{x})=\mathrm{x} / 2$ and $\mathrm{x}_{\mathrm{n}+1}=\mathrm{f}\left(\mathrm{x}_{\mathrm{n}}\right)$, we have $x_{n}=x / 2^{n}$. Since, $b(x, y)=2+(x, y)^{2}$, we get $\lim _{n}$, ${ }_{\mathrm{m} \rightarrow \infty} \mathrm{b}\left(\mathrm{x}_{\mathrm{n}}, \mathrm{x}_{\mathrm{m}}\right)=\lim _{\mathrm{n}, \mathrm{m} \rightarrow \infty} 2+\mathrm{x}_{\mathrm{n}}+\mathrm{x}_{\mathrm{m}}=2<8=1 / \lambda$. The sequence $\left\{x_{n}\right\}=\left\{x / 2^{n}\right\}$ is convergent to 0 that is $\lim _{n, m-\infty} d_{b}\left(x_{n}\right.$, $0)=\lim _{n, m \rightarrow \infty} d_{b}\left(x / 2^{n}, 0\right)=\lim _{n \rightarrow \infty}(4)^{32}\left|\frac{x}{2^{n}}\right|=1$. Thus, based on Theorem 3.1, we conclude that $\mathrm{x}^{*}=0$ is a unique fixed point of $f$.

Theorem 3.2: Let $\left(X, d_{b}\right)$ be a complete extended multiplicative b-metric space $\mathrm{f}: \mathrm{X} \rightarrow \mathrm{X}$ and be a mapping that satisfies the following conditions:

$$
\left(d_{b}(f x, f y)\right)^{b(x, y)} \leq d_{b}^{\lambda}(x, y)
$$

where $0<\lambda<1$. If $\lim _{\mathrm{n}, \mathrm{m} \rightarrow \infty} \mathrm{b}\left(\mathrm{x}_{\mathrm{n}}, \mathrm{x}_{\mathrm{m}}\right)<\frac{1}{\lambda}$, then $\mathrm{f}$ has a fixed point.

Proof: Let taking $\mathrm{x}_{0} \in \mathrm{X}$ and let $\left\{\mathrm{x}_{\mathrm{n}}\right\}$ be a sequence where $\mathrm{x}_{\mathrm{n}}=\mathrm{f}\left(\mathrm{x}_{\mathrm{n}-1}\right)$ for $\mathrm{n}=1,2,3, \ldots$ By using Eq. 8 , we have:

$$
\left(d_{b}\left(x_{n}, x_{n+1}\right)\right)^{b\left(x_{n-1}, x_{n}\right)}=\left(d_{b}\left(f_{n-1}, f_{n}\right)\right)^{b\left(x_{n-1}, x_{n}\right)} \leq\left(d_{b}\left(x_{n-1}, x_{n}\right)\right)^{\lambda}
$$

Since, $d_{b}\left(x_{n-1}, x_{n}\right) \geq 1$ and $b\left(x_{n-1}, x_{n}\right) \geq 1$ by using (3.8), we have:

$$
\left(\mathrm{d}_{\mathrm{b}}\left(\mathrm{x}_{\mathrm{n}}, \mathrm{x}_{\mathrm{n}+1}\right)\right) \leq\left(\mathrm{d}_{\mathrm{b}}\left(\mathrm{x}_{\mathrm{n}-1}, \mathrm{x}_{\mathrm{n}}\right)\right)^{\frac{\lambda}{\mathrm{b}\left(\mathrm{x}_{\mathrm{n}-1}, \mathrm{x}_{\mathrm{n}}\right)}} \leq\left(\mathrm{d}_{\mathrm{b}}\left(\mathrm{x}_{\mathrm{n}-1}, \mathrm{x}_{\mathrm{n}}\right)\right)^{\lambda}
$$

Recursively, we have $d_{b}\left(x_{n}, x_{n+1}\right) \leq d_{b}^{\lambda n}\left(x_{0}, x_{1}\right)$. Since, $0<\lambda<1$ we have $d_{b}\left(x_{n}, x_{n+1}\right) \rightarrow 1$ as $n \rightarrow \infty$. Next, we have to show that $\left\{\mathrm{x}_{\mathrm{n}}\right\}$ is a Cauchy sequence. Consider that for all $\mathrm{n}, \mathrm{m} \in \mathrm{N}$ with $\mathrm{m}>\mathrm{n}$ by the multiplicative triangle inequality in Eq. 1, we have:

$$
\begin{aligned}
& d_{b}\left(x_{n}, x_{m}\right) \leq\left(d_{b}\left(x_{n}, x_{n+1}\right)\right)^{b\left(x_{n}, x_{m}\right)} \times\left(d_{b}\left(x_{n+1}, x_{m}\right)\right)^{b\left(x_{n}, x_{m}\right)} \leq \\
& \left(d_{b}\left(x_{0}, x_{1}\right)\right)^{\lambda^{n} b\left(x_{n}, x_{m}\right)} \times\left(d_{b}\left(x_{n+1}, x_{n+2}\right)\right)^{b\left(x_{n}, x_{m}\right) b\left(x_{n+1}, x_{m}\right)} \times \\
& \left(d_{b}\left(x_{n+2}, x_{m}\right)\right)^{b\left(\left(x_{n}, x_{m}\right) b\left(x_{n+1}, x_{m}\right)\right.} \leq\left(d_{b}\left(x_{0}, x_{1}\right)\right)^{\lambda^{n} b\left(x_{n}, x_{m}\right)} \times \\
& \left(d_{b}\left(x_{0}, x_{1}\right)\right)^{\lambda^{n+1} b\left(x_{n}, x_{m}\right) b\left(\left(x_{n+1}, x_{m}\right)\right.} \times\left(d_{b}\left(x_{n+2}, x_{m}\right)\right)^{b\left(x_{n}, x_{m}\right) b\left(x_{n+1}, x_{m}\right)} \leq \\
& \left(d_{b}\left(x_{0}, x_{1}\right)\right)^{\lambda^{n} b\left(x_{n_{n}}, x_{m}\right)} \times\left(d_{b}\left(x_{0}, x_{1}\right)\right)^{\lambda^{n+b} b\left(x_{n}, x_{m}\right) b\left(x_{n+1}, x_{m}\right)} \times \\
& \left(d_{b}\left(x_{0}, x_{1}\right)\right)^{\lambda^{m-1} b\left(x_{n}, x_{m}\right) b\left(x_{m-1}, x_{m}\right)}\left(d_{b}\left(x_{0}, x_{1}\right)\right)^{\left(\sum_{j=0}^{m, n-1-1} \lambda^{n+1} \prod_{i=0}^{j} b\left(x_{n+t}, x_{m}\right)\right.}
\end{aligned}
$$

In fact, since, $\mathrm{b}\left(\mathrm{x}_{\mathrm{i}}, \mathrm{x}_{\mathrm{m}}\right) \geq 1$ by using Eq. 10 , we have:

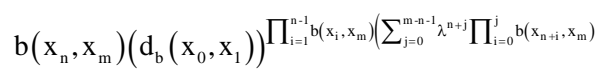

Let:

$$
\mathrm{S}_{\mathrm{n}}=\sum_{\mathrm{j}=1}^{\mathrm{n}} \lambda^{\mathrm{j}} \prod_{\mathrm{i}=1}^{\mathrm{j}} \mathrm{b}\left(\mathrm{x}_{\mathrm{i}}, \mathrm{x}_{\mathrm{m}}\right)
$$

Then by using Eq. 11, we obtain:

$$
\mathrm{d}_{\mathrm{b}}\left(\mathrm{x}_{\mathrm{n}}, \mathrm{x}_{\mathrm{m}}\right) \leq\left(\mathrm{d}_{\mathrm{b}}\left(\mathrm{x}_{0}, \mathrm{x}_{1}\right)\right)^{\mathrm{S}_{\mathrm{m}-1}-\mathrm{S}_{\mathrm{n}-1}}
$$

Let:

$$
\beta_{\mathrm{j}}=\lambda^{\mathrm{j}} \prod_{\mathrm{i}=0}^{\mathrm{j}} \mathrm{b}\left(\mathrm{x}_{\mathrm{i}}, \mathrm{x}_{\mathrm{m}}\right)
$$

We have:

$$
\mathrm{S}_{\mathrm{n}}=\sum_{\mathrm{j}=1}^{\mathrm{n}} \beta_{\mathrm{j}}
$$

By using the ratio test and since, $\lim _{n, m \rightarrow \infty} b\left(x_{n}, x_{m}\right)<\frac{1}{\lambda}$, we have $\operatorname{Lim}_{j \rightarrow \infty} \frac{\beta j}{\beta_{i-1}}=\lim _{j \rightarrow \infty} \lambda b\left(x_{j}, x_{m}\right)=\lambda \lim _{j, m \rightarrow \infty} b\left(x_{j}, x_{m}\right)<1$ for each $m \in N$. Thus, we have $\left\{S_{n}\right\}$ is convergent for each $m \in N$, this implies $\lim _{\mathrm{n}, \mathrm{m}-\infty} \mathrm{S}_{\mathrm{m}-\mathrm{1}}-\mathrm{S}_{\mathrm{n}-1}=0$.

Then by using Eq. 12 , we get $d_{b}\left(x_{n}, x_{n}\right) \rightarrow 1$ as $n, m \rightarrow \infty$. Hence, $\left\{S_{n}\right\}$ is a Cauchy sequence. Since, $X$ is complete, there exists $x^{*} \in X$ such that $d_{b}\left(x_{n}, x^{*}\right) \rightarrow 1$ as $n \rightarrow \infty$. Claim that $\mathrm{x}^{*}$ is the fixed point of $\mathrm{f}$. Consider that:

$$
\begin{aligned}
& d_{b}\left(f^{*}, x^{*}\right) \leq\left(d_{b}\left(f x^{*}, x_{n+1}\right)\right)^{b\left(f x^{*}, x^{*}\right)} \times\left(d_{b}\left(x_{n+1}, x^{*}\right)\right)^{b\left(f x^{*}, x^{*}\right)}= \\
& \left(d_{b}\left(f^{*}, f_{x}\right)\right)^{b\left(f x^{*}, x^{*}\right)} \times\left(d_{b}\left(x_{n+1}, x^{*}\right)\right)^{b\left(f x^{*}, x^{*}\right)}= \\
& \left(\left(d_{b}\left(x_{n+1}, x^{*}\right)\right)^{\lambda}\right)^{b} \frac{b\left(x^{*}, x^{*}\right)}{b\left(x^{*}, x_{n}\right)} \times\left(d_{b}\left(x_{n+1}, x^{*}\right)\right)^{b\left(f x^{*}, x^{*}\right)} \leq
\end{aligned}
$$

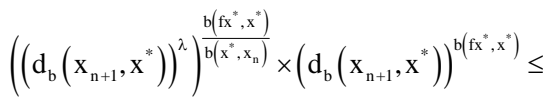

$$
\begin{aligned}
& \left(\left(d_{b}\left(x_{n+1}, x^{*}\right)\right)^{\lambda}\right)^{b\left(x^{*}, x^{*}\right)} \times\left(d_{b}\left(x_{n+1}, x^{*}\right)\right)^{b\left(f x^{*}, x^{*}\right)}
\end{aligned}
$$

Since, $d_{b}\left(x_{n}, x^{*}\right) \rightarrow 1$ as $n \rightarrow \infty$ then from Eq. 13, we get $d_{b}\left(f^{*}, x^{*}\right)=1$ that is $\mathrm{fx}^{*}=\mathrm{x}^{*}$. Hence, $\mathrm{x}^{*}$ is the fixed point of $f$. For uniqueness of the fixed point, suppose $u^{*}$ is another fixed point of $\mathrm{f}$, that is $\mathrm{fu}^{*}=\mathrm{u}^{*}$. Consider that $d_{b}\left(u^{*}, x^{*}\right)=d_{b}\left(f^{*}, f x^{*}\right) \leq\left(d_{b}\left(u^{*}, x^{*}\right)\right)^{\lambda\left(u^{*}, x^{*}\right)} \leq\left(d_{b}\left(u^{*}, x^{*}\right)\right)^{\lambda}$. Thus, we get $\left(d_{b}\left(u^{*}, x^{*}\right)\right)^{1-\lambda} \leq 1$. Since, $1-\lambda>0$ and $d_{b}\left(u^{*}\right.$, $\left.x^{*}\right) \geq 1$, we get $d_{b}\left(u^{*}, x^{*}\right)=1$ that is $u^{*}=x^{*}$.

Example 3.2: As shown in Example 2.4 by taking a $=4^{32}$ that is $b(x, y)=2+(x-y)^{2}$ and $d_{b}(x, y)=(4)^{32|x-y| 2}$ where $x$, $y \in X=[0,1]$. Let $f(x)=x^{4} / 2^{4}$, for $x, y \in X$. Let $x \neq y$, consider that:

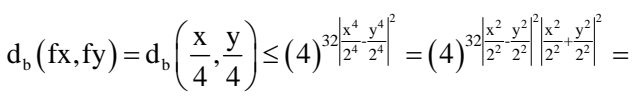

$$
\begin{aligned}
& (4)^{\frac{32}{4}\left|x^{2}-y^{2}\right| \mid \frac{x^{2}}{2^{2}} \frac{1}{2}^{2} \frac{2}{2}^{2}} \leq(4)^{\frac{32}{4}\left|x^{2}-y^{2}\right|^{2}(2)} \leq(4)^{\frac{32}{4}\left|x^{2}-y^{2}\right|\left(2+(x-y)^{2}\right)}= \\
& \left((4)^{32|x-y|^{2}}\right)^{\frac{1}{4}\left(2+(x-y)^{2}\right)}=\left(d_{b}(x, y)\right)^{\lambda b(x, y)}
\end{aligned}
$$

Hence, we obtain:

$$
\left(d_{b}(f x, f y)\right)^{b(x, y)} \leq\left(d_{b}(x, y)\right)^{\lambda}
$$


where, $\lambda=1 / 4<1$. By using $\mathrm{f}(\mathrm{x})=\mathrm{x}^{4} / 2^{4}$ and $\mathrm{x}_{\mathrm{n}+1}=\mathrm{f}\left(\mathrm{x}_{\mathrm{n}}\right)$ with $\mathrm{x}_{0}=\mathrm{x}$, we have $\mathrm{x}_{\mathrm{n}}=\alpha^{\frac{4^{\mathrm{n}}-1}{3}} \mathrm{x}^{4^{\mathrm{n}}}$ where $\alpha=1 / 2^{4}$. By using $\mathrm{b}(\mathrm{x}, \mathrm{y})=2+(\mathrm{x}-\mathrm{y})^{2}$, we have:

$$
\begin{aligned}
& \lim _{n, m \rightarrow \infty} b\left(x_{n}, x_{m}\right)=\lim _{n, m \rightarrow \infty} 2+x_{n}+x_{m}=\lim _{n, m \rightarrow \infty} 2+\alpha^{\frac{4^{n}-1}{3}} x^{4^{n}}+ \\
& \alpha^{\frac{4^{-m}-1}{3}} x^{4^{m}}=2<4=\frac{1}{\lambda}
\end{aligned}
$$

Since, $\alpha=1 / 2^{4}<1$ and $\mathrm{x} \in[0,1]$, the sequence $\left\{x_{n}\right\}=\left\{\alpha^{\frac{4^{n}-1}{3} x^{4^{n}}}\right\}$ is convergent to 0 . Indeed:

$$
\begin{aligned}
& \lim _{n \rightarrow \infty} d_{b}\left(x_{n}, 0\right)=\lim _{n \rightarrow \infty} d_{b}\left(a^{\frac{4^{n}-1}{3}} x^{4^{n}}, 0\right)= \\
& \lim _{n \rightarrow \infty}(4)^{32}\left(a^{\frac{4^{n} \cdot-1}{3} x^{x^{n}}}\right)^{2}=1
\end{aligned}
$$

Thus, based on Theorem 3.2, we conclude that $\mathrm{x}^{*}=$ 0 is a unique fixed point of $f$.

Theorem 3.3: Let $\left(X, d_{b}\right)$ be a complete extended multiplicative b-metric space and $f: X \rightarrow X$ be a mapping that satisfies the following conditions:

$$
\left(d_{b}(f x, f y)\right)^{b(x, y)} \leq d_{b}^{\lambda}(x, f x) \times d_{b}^{\gamma}(y, f y) \times \frac{d_{b}^{\beta}(x, f y) \times d_{b}^{\beta}(y, f x)}{1+d_{b}^{\beta}(x, f y) \times d_{b}^{\beta}(y, f x)^{\prime}}
$$

where $0 \leq \beta, \quad \gamma<1, \quad 0<\lambda<1$ and $0<\lambda / 1-\gamma<1$. If $\lim _{\mathrm{n}, \mathrm{m} \rightarrow \infty} \mathrm{b}\left(\mathrm{x}_{\mathrm{n}}, \mathrm{x}_{\mathrm{m}}\right)<\frac{1-\gamma}{\lambda}$, then $\mathrm{f}$ has a fixed point.

Proof: Let taking $\mathrm{x}_{\mathrm{o}} \in \mathrm{X}$ and let $\left\{\mathrm{x}_{\mathrm{n}}\right\}$ be a sequence where $\mathrm{x}_{\mathrm{n}}=\mathrm{f}\left(\mathrm{x}_{\mathrm{n}-1}\right)$ for $\mathrm{n}=1,2,3, \ldots$. By using Eq. 14, we have:

$$
\begin{aligned}
& \mathrm{d}_{\mathrm{b}}\left(\mathrm{x}_{\mathrm{n}}, \mathrm{x}_{\mathrm{n}+1}\right)=\mathrm{d}_{\mathrm{b}}\left(\mathrm{fx}_{\mathrm{n}-1}, \mathrm{fx}_{\mathrm{n}}\right) \leq \\
& \left(\begin{array}{l}
d_{b}^{\lambda}\left(x_{n-1}, f x_{n-1}\right) \times d_{b}^{\gamma}\left(x_{n}, f x_{n}\right) \times \\
\frac{d_{b}^{\beta}\left(x_{n-1}, f x_{n}\right) \times d_{b}^{\beta}\left(x_{n}, f x_{n-1}\right)}{1+d_{b}^{\beta}\left(x_{n-1}, f x_{n}\right)+d_{b}^{\beta}\left(x_{n}, f x_{n-1}\right)}
\end{array}\right)^{\frac{1}{b\left(x_{n-1}, x_{n}\right)}}= \\
& \left(\begin{array}{l}
d_{b}^{\lambda}\left(x_{n-1}, x_{n}\right) \times d_{b}^{\gamma}\left(x_{n}, x_{n+1}\right) \times \\
\frac{d_{b}^{\beta}\left(x_{n-1}, x_{n+1}\right) \times d_{b}^{\beta}\left(x_{n}, x_{n}\right)}{1+d_{b}^{\beta}\left(x_{n-1}, x_{n+1}\right)+d_{b}^{\beta}\left(x_{n}, x_{n}\right)}
\end{array}\right)^{\frac{1}{b\left(x_{n-1}, x_{n}\right)}}= \\
& \left(\begin{array}{l}
d_{b}^{\lambda}\left(x_{n-1}, x_{n}\right) \times d_{b}^{\gamma}\left(x_{n}, x_{n+1}\right) \times \\
\frac{d_{b}^{\beta}\left(x_{n-1}, x_{n+1}\right)}{2+d_{b}^{\beta}\left(x_{n-1}, x_{n+1}\right)}
\end{array}\right)^{\frac{1}{b\left(x_{n-1}, x_{n}\right)}} \leq\left(\begin{array}{l}
\left.d_{b}^{\lambda}\left(x_{n-1}, x_{n}\right) \times\right)^{\frac{1}{b\left(x_{n-1}, x_{n}\right)}} \leq \\
d_{b}^{\gamma}\left(x_{n}, x_{n+1}\right)
\end{array}\right)^{-} \\
& \left(d_{b}^{\lambda}\left(x_{n-1}, x_{n}\right) \times d_{b}^{\gamma}\left(x_{n}, x_{n+1}\right)\right)^{\frac{1}{b\left(x_{n-1}, x_{n}\right)}}=\left(d_{b}\left(x_{n-1}, x_{n}\right)\right)^{\frac{\lambda}{b\left(x_{n-1}, x_{n}\right)}} \times \\
& \left(d_{b}\left(x_{n}, x_{n+1}\right)\right)^{\frac{\gamma}{b\left(x_{n-1}, x_{n}\right)}} \leq\left(d_{b}\left(x_{n-1}, x_{n}\right)\right)^{\lambda} \times\left(d_{b}\left(x_{n}, x_{n+1}\right)\right)^{\gamma}
\end{aligned}
$$

Thus, we get:

$$
d_{b}\left(x_{n}, x_{n+1}\right) \leq\left(d_{b}\left(x_{n-1}, x_{n}\right)\right)^{\frac{\lambda}{1-\gamma}}
$$

Let $\delta=\lambda / 1-\gamma^{\prime}$ recursively, we have:

$$
d_{b}\left(x_{n}, x_{n+1}\right) \leq d_{b}^{\delta^{n}}\left(x_{0}, x_{1}\right)
$$

Since, $0<\delta<1$, so, we get $d_{b}\left(x_{n}, x_{n+1}\right) \rightarrow 1$ as $n \rightarrow \infty$. Next, we have to show that $\left\{x_{n}\right\}$ is a Cauchy sequence. Consider that for all $n, m \in N$ with by $m>n$ the multiplicative triangle inequality in Eq. 1, we have:

$$
\begin{aligned}
& d_{b}\left(x_{n}, x_{m}\right) \leq\left(d_{b}\left(x_{n}, x_{n+1}\right)\right)^{b\left(x_{n}, x_{m}\right)} \times\left(d_{b}\left(x_{n+1}, x_{m}\right)\right)^{b\left(x_{n}, x_{m}\right)} \leq \\
& \left(d_{b}\left(x_{0}, x_{1}\right)\right)^{\delta^{n} b\left(x_{n}, x_{m}\right)} \times\left(d_{b}\left(x_{n+1}, x_{n+2}\right)\right)^{b\left(x_{n}, x_{m}\right) b\left(x_{n+1}, x_{m}\right)} \times \\
& \left(d_{b}\left(x_{n+2}, x_{m}\right)\right)^{b\left(\left(x_{n}, x_{m}\right) b\left(x_{n_{1}+1}, x_{m}\right)\right.} \leq\left(d_{b}\left(x_{0}, x_{1}\right)\right)^{\delta^{n} b\left(x_{n}, x_{m}\right)} \times \\
& \left(d_{b}\left(x_{0}, x_{1}\right)\right)^{\delta^{n+1} b\left(x_{n}, x_{m}\right) b\left(\left(x_{n+1}, x_{m}\right)\right.} \times\left(d_{b}\left(x_{n+2}, x_{m}\right)\right)^{b\left(x_{n}, x_{m}\right) b\left(x_{n+1}, x_{m}\right)} \leq \\
& \left(\mathrm{d}_{\mathrm{b}}\left(\mathrm{x}_{0}, \mathrm{x}_{1}\right)\right)^{\delta^{\mathrm{n}} \mathrm{b}\left(\mathrm{x}_{\mathrm{n}}, \mathrm{x}_{\mathrm{m}}\right)} \times\left(\mathrm{d}_{\mathrm{b}}\left(\mathrm{x}_{0}, \mathrm{x}_{1}\right)\right)^{\delta^{n^{n+}} \mathrm{b}\left(\mathrm{x}_{\mathrm{n}}, \mathrm{x}_{\mathrm{m}}\right) \mathrm{b}\left(\left(\mathrm{x}_{\mathrm{n}+1}, \mathrm{x}_{\mathrm{m}}\right)\right.} \times \ldots \times
\end{aligned}
$$

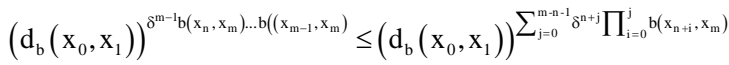

In fact, since, $\mathrm{b}\left(\mathrm{x}_{\mathrm{i}}, \mathrm{x}_{\mathrm{m}}\right) \geq 1$ by using Eq. 16, we have:

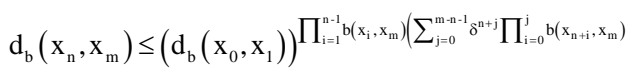

Let $\mathrm{U}_{\mathrm{n}}=\sum_{\mathrm{j}=1}^{\mathrm{n}} \delta^{\mathrm{j}} \prod_{\mathrm{i}=1}^{\mathrm{j}} \mathrm{b}\left(\mathrm{x}_{\mathrm{i}}, \mathrm{x}_{\mathrm{m}}\right)$ by using Eq. 17, we obtain:

$$
d_{b}\left(x_{n}, x_{m}\right) \leq\left(d_{b}\left(x_{0}, x_{1}\right)\right)^{\mathrm{U}_{m-1}-U_{n-1}}
$$

Let $\sigma_{j}=\delta^{j} \prod_{i=0}^{j} b\left(x_{i}, x_{m}\right)$, we have $U_{n}=\sum_{j=1}^{n} \sigma^{j}$. By using the ratio test and since, $\lim _{n, m \rightarrow \infty} b\left(x_{n}, x_{m}\right)<\frac{1-\gamma}{\lambda}$, we have $\operatorname{Lim}_{j \rightarrow \infty} \frac{\sigma j}{\sigma_{j-1}}=\lim _{j \rightarrow \infty} \delta b\left(x_{j}, x_{m}\right)=\delta \lim _{j, m \rightarrow \infty} b\left(x_{j}, x_{m}\right)<\delta\left(\frac{1-\gamma}{\lambda}\right)=1$ for each $m \in N$. Thus, we have $\left\{U_{n}\right\}$ is convergent for each $\mathrm{m} \in \mathrm{N}$, this implies $\lim _{\mathrm{n}, \mathrm{m}-\infty} \mathrm{U}_{\mathrm{m}-1}-\mathrm{U}_{\mathrm{n}-1}=0$. Then by using Eq. 18, we get $d_{b}\left(x_{n}, x_{m}\right) \rightarrow 1$ as $n, m \rightarrow \infty$.

Hence, $\left\{x_{n}\right\}$ is a Cauchy sequence. Since, $X$ is complete, there exists $x^{*} \in X$ such that $d_{b}\left(x_{n}, x^{*}\right) \rightarrow 1$ as $n \rightarrow \infty$. Claim that is the fixed point of . Consider that from Eq. 1 and 14, we have:

$$
\begin{aligned}
& d_{b}\left(f x^{*}, x^{*}\right) \leq\left(d_{b}\left(f x^{*}, x_{n+1}\right)\right)^{b\left(f x^{*}, x^{*}\right)} \times\left(d_{b}\left(x_{n+1}, x^{*}\right)\right)^{b\left(f x^{*}, x^{*}\right)}= \\
& \left.\left(d_{b}\left(f x^{*}, f x_{n}\right)\right)^{b\left(x^{*}, x^{*}\right)} \times\left(d_{b}\left(x_{n+1}, x^{*}\right)\right)^{b\left(x^{*}, x^{*}\right)}=\left(d_{b}\left(x^{*}, f x^{*}\right)\right)\right)^{\frac{\lambda b\left(x^{*}, x^{*}\right)}{b\left(x_{n}\right)} \times} \times \\
& \left.\left(d_{b}\left(x_{n}, f x_{n}\right)\right)\right)^{\left.\frac{\gamma b\left(x^{*}, x^{*}\right)}{b}, x_{n}\right)} \times\left(\frac{d_{b}^{\beta}\left(x^{*}, f x_{n}\right) \cdot d_{b}^{\beta}\left(x_{n}, f x^{*}\right)}{1+d_{b}^{\beta}\left(x^{*}, f x_{n}\right)+d_{b}^{\beta}\left(x_{n}, f x^{*}\right)}\right)^{\frac{\beta b}{b\left(x^{*}, x^{*}, x_{n}\right)}} \times
\end{aligned}
$$




$$
\begin{aligned}
& \left(d_{b}\left(x_{n+1}, x^{*}\right)\right)^{b\left(f x^{*}, x^{*}\right)}=\left(d_{b}\left(x^{*}, f x^{*}\right)\right)^{\left.\frac{\gamma b\left(f\left(x^{*}, x^{*}\right)\right.}{b}, x_{n}\right)} \times\left(d_{b}\left(x_{n}, f x_{n}\right)\right)^{\frac{\gamma b\left(x^{*}, x^{*}\right)}{b\left(x^{*}, x_{n}\right)}} \times \\
& \left(d_{b}^{\beta}\left(x^{*}, f x_{n}\right)\right)^{\frac{\beta b\left(f^{*}, x^{*}\right)}{b\left(x^{*}, x_{n}\right)}} \times\left(d_{b}\left(x_{n+1}, x^{*}\right)\right)^{b\left(f x^{*}, x^{*}\right)}=\left(d_{b}\left(x^{*}, f x^{*}\right)\right)^{\frac{\lambda b\left(f^{*}, x^{*}\right)}{b\left(x^{*}, x_{n}\right)}} \times \\
& \left(d_{b}\left(x_{n}, x_{n+1}\right)\right)^{\frac{\gamma b\left(x^{*}, x^{*}\right)}{b\left(x^{*}, x_{n}\right)}} \times\left(d_{b}^{\beta}\left(x^{*}, x_{n+1}\right)\right)^{\frac{\beta b\left(f x^{*}, x^{*}\right)}{b\left(x^{*}, x_{n}\right)}} \times\left(d_{b}\left(x_{n+1}, x^{*}\right)\right)^{b\left(f^{*}, x^{*}\right)} \leq \\
& \left(d_{b}\left(x^{*}, f x^{*}\right)\right)^{\lambda b\left(f x^{*}, x^{*}\right)} \times\left(d_{b}\left(x_{n}, x_{n+1}\right)\right)^{\gamma b\left(x^{*}, x^{*}\right)} \times\left(d_{b}^{\beta}\left(x^{*}, x_{n+1}\right)\right)^{b\left(f^{*}, x^{*}\right)} \times \\
& \left(d_{b}\left(x_{n+1}, x^{*}\right)\right)^{b\left(f^{*}, x^{*}\right)}
\end{aligned}
$$

So, from Eq. 19, we get:

$$
\begin{aligned}
& \left(d_{b}\left(f x^{*}, x^{*}\right)\right)^{1-\lambda b\left(f x^{*}, x^{*}\right)} \leq\left(d_{b}\left(x_{n}, x_{n+1}\right)\right)^{\gamma b\left(f x^{*}, x^{*}\right)} \times \\
& \left(d_{b}\left(x_{n+1}, x^{*}\right)\right)^{b\left(f x^{*}, x^{*}\right)} \times\left(d_{b}^{\beta}\left(x^{*}, x_{n+1}\right)\right)^{b\left(f x^{*}, x^{*}\right)}
\end{aligned}
$$

By using Eq. 15 and 20, we get:

$$
\begin{aligned}
& \left(d_{b}\left(f x^{*}, x^{*}\right)\right)^{1-\lambda b\left(f x^{*}, x^{*}\right)} \leq\left(d_{b}^{\delta^{n}}\left(x_{0}, x_{1}\right)\right)^{\gamma b\left(\mathrm{x}^{*}, x^{*}\right)} \times \\
& \left(d_{b}^{\beta}\left(x^{*}, x_{n+1}\right)\right)^{\beta b\left(f x^{*}, x^{*}\right)} \times\left(d_{b}\left(x_{n+1}, x^{*}\right)\right)^{b\left(f x^{*}, x^{*}\right)}= \\
& \left(d_{b}\left(x_{0}, x_{1}\right)\right)^{\gamma \delta^{n} b\left(f x^{*}, x^{*}\right)} \times\left(d_{b}^{\beta}\left(x^{*}, x_{n+1}\right)\right)^{\beta b\left(f x^{*}, x^{*}\right)} \times \\
& \left(d_{b}\left(x_{n+1}, x^{*}\right)\right)^{b\left(f x^{*}, x^{*}\right)}
\end{aligned}
$$

Thus, we get:

$$
\begin{aligned}
& d_{b}\left(f x^{*}, y^{*}\right) \leq\left(d_{b}\left(x_{0}, x_{1}\right)\right)^{\frac{\gamma \delta^{n} b\left(f x^{*}, x^{*}\right)}{1-\lambda b\left(f x^{*}, x^{*}\right)}} \times\left(d_{b}^{\beta}\left(x^{*}, x_{n+1}\right)\right)^{\left.\frac{\beta b}{1-\lambda b\left(f x^{*}, x^{*}\right)} x^{*} x^{*}\right)} \times \\
& \left(d_{b}\left(x_{n+1}, x^{*}\right)\right)^{\frac{b\left(f x^{*}, x^{*}\right)}{1-\lambda\left(x^{*}, x^{*}\right)}}
\end{aligned}
$$

Since, $\delta^{n} \rightarrow 0$ and $d_{b}\left(x_{n}, x^{*}\right) \rightarrow 1$ as $n \rightarrow \infty$ then from Eq. 21 we get $d_{b}\left(f x^{*}, x^{*}\right) \leq 1$. Since, $d_{b}\left(f x^{*}, x^{*}\right) \geq 1$, then we have $d_{b}\left(f x^{*}, x^{*}\right)=1$ that is $f x^{*}=x^{*}$. Hence, $x^{*}$ is the fixed point of $f$. For uniqueness of the fixed point, suppose $\mathrm{u}^{*}$ is another fixed point of $\mathrm{f}$ that is $\mathrm{fu}^{*}=\mathrm{u}^{*}$. By using Eq. 14 we have:

$$
\begin{aligned}
& \left(d_{b}\left(u^{*}, x^{*}\right)\right)=\left(d_{b}\left(f u^{*}, f x^{*}\right)\right) \leq\left(d_{b}\left(u^{*}, f u^{*}\right)\right)^{\frac{\lambda}{b\left(u^{*}, x^{*}\right)}} \times \\
& \left(d_{b}\left(x^{*}, f x^{*}\right)\right)^{\frac{\gamma}{b\left(u^{*}, x^{*}\right)}} \times\left(\frac{d_{b}\left(u^{*}, f x^{*}\right)^{\beta} \times\left(d_{b}\left(x^{*}, f u^{*}\right)\right)^{\beta}}{1+d_{b}^{\beta}\left(u^{*}, f x^{*}\right)+d_{b}^{\beta}\left(x^{*}, f u^{*}\right)}\right)^{\frac{1}{b\left(u^{*}, x^{*}\right)}}= \\
& \left(d_{b}\left(u^{*}, u^{*}\right)\right)^{\frac{\lambda}{b\left(u^{*}, x^{*}\right)} \times\left(d_{b}\left(x^{*}, x^{*}\right)\right) \frac{\gamma}{b\left(u^{*}, x^{*}\right)} \times} \\
& \left(\frac{d_{b}\left(u^{*}, x^{*}\right)^{\beta} \times\left(d_{b}\left(x^{*}, u^{*}\right)\right)^{\beta}}{1+d_{b}^{\beta}\left(u^{*}, x^{*}\right)+d_{b}^{\beta}\left(x^{*}, u^{*}\right)}\right)^{\frac{1}{b\left(u^{*}, x^{*}\right)}}= \\
& \left(\frac{d_{b}\left(u^{*}, x^{*}\right)^{\beta} \times\left(d_{b}\left(x^{*}, u^{*}\right)\right)^{\beta}}{1+d_{b}^{\beta}\left(u^{*}, x^{*}\right)+d_{b}^{\beta}\left(x^{*}, u^{*}\right)}\right)^{\frac{1}{b\left(u^{*}, x^{*}\right)}} \leq\left(d_{b}\left(u^{*}, x^{*}\right)\right)^{\frac{\beta}{b\left(u^{*}, x^{*}\right)}} \leq \\
& \left(d_{b}\left(u^{*}, x^{*}\right)\right)^{\beta} \\
& (2)
\end{aligned}
$$

Thus, from Eq. 22, we get $\left(d_{b}\left(u^{*}, x^{*}\right)\right)^{1-\beta} \leq 1$. Since, $1-\beta \geq 0$ and $d_{b}\left(u^{*}, x^{*}\right) \geq 1$, so we get $d_{b}\left(u^{*}, x^{*}\right)=1$. This implies that $\mathrm{u}^{*}=\mathrm{x}^{*}$.

Theorem 3.4: Let $\left(X, d_{b}\right)$ be a complete extended multiplicative b-metric space and $\mathrm{f}, \mathrm{g}: \mathrm{X} \rightarrow \mathrm{X}$ be two mappings such that $\mathrm{fX} \subseteq \mathrm{gX}$, gX closed and satisfying the following conditions:

$$
\left(d_{b}(f x, f y)\right)^{b(x, y)} \leq d_{b}^{\beta}(f x, g x) \times d_{b}^{\gamma(x, y)}(f y, g y) d_{b}^{\lambda}(g x, g y)
$$

where $\quad \beta \geq 0, \quad 0 \leq \gamma<1, \quad 0<\lambda<1, \quad 0<\beta+\lambda /(1-\gamma)<1 \quad$ and $\lim _{\mathrm{n}, \mathrm{m} \rightarrow \infty} \mathrm{b}\left(\mathrm{y}_{\mathrm{n}}, \mathrm{y}_{\mathrm{m}}\right)<\frac{1-\gamma}{\beta+\lambda}$. If $\mathrm{f}$, g weakly compatible, then $\mathrm{f}, \mathrm{g}$ have a unique common fixed point.

Proof: Let $\mathrm{x}_{0} \in \mathrm{X}$. Since, $\mathrm{fX} \subseteq \mathrm{gX}$, we can define two sequences $\left\{x_{n}\right\}$ and $\left\{y_{n}\right\}$ where $y_{n}=f_{n}=g x_{n+1}$. By using Eq. 23, we have:

$$
\begin{aligned}
& \left(d_{b}\left(y_{n}, y_{n+1}\right)\right)^{b\left(x_{n}, x_{n+1}\right)}=\left(d_{b}\left(f x_{n}, f x_{n+1}\right)\right)^{b\left(x_{n}, x_{n+1}\right)} \leq d_{b}^{\beta}\left(f x_{n}, g x_{n}\right) \times \\
& d_{b}^{\gamma b\left(x_{n}, x_{n+1}\right)}\left(f x_{n+1}, g x_{n+1}\right) \times d_{b}^{\lambda}\left(g x_{n}, g x_{n+1}\right)=d_{b}^{\beta+\lambda}\left(y_{n}, y_{n-1}\right) \times \\
& d_{b}^{\gamma b\left(x_{n}, x_{n+1}\right)}\left(y_{n+1}, y_{n}\right) \times d_{b}^{\lambda}\left(y_{n-1}, y_{n}\right)=d_{b}^{\beta+\lambda}\left(y_{n}, y_{n-1}\right) \times \\
& d_{b}^{\gamma b\left(x_{n}, x_{n+1}\right)}\left(y_{n+1}, y_{n}\right)
\end{aligned}
$$

Since, $d_{b}\left(y_{n-1}, y_{n}\right) \geq 1$ and $b\left(x_{n}, x_{n+1}\right) \geq 1$. So, we get:

$$
d_{b}\left(y_{n}, y_{n+1}\right) \leq\left(d_{b}\left(y_{n-1}, y_{n}\right)\right)^{\frac{\beta+\lambda}{b\left(x_{n}, x_{n+1}\right)(1-\gamma)}} \leq\left(d_{b}\left(y_{n-1}, y_{n}\right)\right)^{\frac{\beta+\lambda}{(1-\gamma)}}
$$

Recursively, we have:

$$
d_{b}\left(y_{n}, y_{n+1}\right) \leq\left(d_{b}\left(y_{0}, y_{1}\right)\right)^{\rho^{n}}
$$

where $\rho=\beta+\lambda /(1-\gamma)$. Since, $0<\beta+\lambda /(1-\gamma)$ and using Eq. 24, we have:

$$
\mathrm{d}_{\mathrm{b}}\left(\mathrm{y}_{\mathrm{n}}, \mathrm{y}_{\mathrm{n}+1}\right) \rightarrow 1 \text {, as } \mathrm{n} \rightarrow \infty
$$

Next, we have to show that $\left\{\mathrm{y}_{\mathrm{n}}\right\}$ is a Cauchy sequence. Consider that for all $n, m \in N$ with $m>n$ by the multiplicative triangle inequality in Eq. 1, we have:

$$
\begin{aligned}
& d_{b}\left(y_{n}, y_{m}\right) \leq\left(d_{b}\left(y_{n}, y_{n+1}\right)\right)^{b\left(y_{n}, y_{m}\right)} \times\left(d_{b}\left(y_{n+1}, y_{m}\right)\right)^{b\left(y_{n}, y_{m}\right)} \leq \\
& \left(d_{b}\left(y_{0}, y_{1}\right)\right)^{\rho^{n} b\left(y_{n}, y_{m}\right)} \times\left(d_{b}\left(y_{n+1}, y_{n+2}\right)\right)^{b\left(y_{n}, y_{m}\right) b\left(y_{n+1}, y_{m}\right)} \times \\
& \left(d_{b}\left(y_{n+2}, y_{m}\right)\right)^{b\left(\left(y_{n}, y_{m}\right) b\left(y_{n+1}, y_{m}\right)\right.} \leq\left(d_{b}\left(y_{0}, y_{1}\right)\right)^{\rho^{n} b\left(y_{n}, y_{m}\right)} \times \\
& \left(d_{b}\left(y_{0}, y_{1}\right)\right)^{p^{n+1} b\left(y_{n}, y_{m}\right) b\left(y_{n+1}, y_{m}\right)} \times\left(d_{b}\left(y_{n+2}, y_{m}\right)\right)^{b\left(y_{n}, y_{m}\right) b\left(y_{n+1}, y_{m}\right)} \leq \\
& \left(d_{b}\left(y_{0}, y_{1}\right)\right)^{p^{n} b\left(y_{n}, y_{m}\right)} \times\left(d_{b}\left(y_{0}, y_{1}\right)\right)^{\rho^{n+1} b\left(y_{n}, y_{m}\right) b\left(y_{n+1}, y_{m}\right)} \times \ldots \times \\
& \left(d_{b}\left(y_{0}, y_{1}\right)\right)^{p^{m-1} b\left(y_{n}, y_{m}\right) \ldots b\left(y_{m-1}, y_{m}\right) \leq}\left(d_{b}\left(y_{0}, y_{1}\right)\right)^{\sum_{j=0}^{m-n-1} \rho^{n+j} \prod_{i=0}^{j} b\left(y_{n+1}, y_{m}\right)}
\end{aligned}
$$

In fact, since, $b\left(y_{i}, y_{m}\right) \geq 1$ by using Eq. 26, we have: 


$$
\mathrm{d}_{\mathrm{b}}\left(\mathrm{y}_{\mathrm{n}}, \mathrm{y}_{\mathrm{m}}\right) \leq\left(\mathrm{d}_{\mathrm{b}}\left(\mathrm{y}_{0}, \mathrm{y}_{1}\right)\right)^{\prod_{\mathrm{i}=1}^{\mathrm{n}-1} \mathrm{~b}\left(\mathrm{y}_{\mathrm{nti}}, \mathrm{y}_{\mathrm{m}}\right) \sum_{\mathrm{j}=0}^{\mathrm{m} \cdot \mathrm{n}-1} \mathrm{\rho}^{\mathrm{n}+j} \prod_{\mathrm{i}=0}^{\mathrm{j}} \mathrm{b}\left(\mathrm{y}_{\mathrm{n}+\mathrm{i}}, \mathrm{y}_{\mathrm{m}}\right)}
$$

Let $\mathrm{W}_{\mathrm{n}}=\sum_{\mathrm{j}=1}^{\mathrm{n}} \rho^{\mathrm{j}} \prod_{\mathrm{i}=1}^{\mathrm{j}} \mathrm{b}\left(\mathrm{y}_{\mathrm{i}}, \mathrm{y}_{\mathrm{m}}\right)$, we obtain:

$$
\mathrm{d}_{\mathrm{b}}\left(\mathrm{y}_{\mathrm{n}}, \mathrm{y}_{\mathrm{m}}\right) \leq\left(\mathrm{d}_{\mathrm{b}}\left(\mathrm{y}_{0}, \mathrm{y}_{1}\right)\right)^{\mathrm{w}_{\mathrm{m}-1}-\mathrm{w}_{\mathrm{n}-1}}
$$

Let $\gamma_{\mathrm{j}}=\rho^{\mathrm{j}} \prod_{\mathrm{i}=0}^{\mathrm{j}} \mathrm{b}\left(\mathrm{y}_{\mathrm{i}}, \mathrm{y}_{\mathrm{m}}\right)$, we have $\mathrm{W}_{\mathrm{n}}=\sum_{\mathrm{j}=1}^{\mathrm{n}} \gamma \mathrm{j}$. By using the ratio test and since:

$$
\lim _{n, m \rightarrow \infty} b\left(y_{n}, y_{m}\right)<\frac{1-\gamma}{\beta+\lambda}=\frac{1}{\rho}
$$

we have:

$$
\operatorname{Lim}_{j \rightarrow \infty} \frac{\gamma j}{\gamma_{j-1}}=\lim _{j \rightarrow \infty} \rho b\left(y_{j}, y_{m}\right)=\rho \lim _{j \rightarrow \infty} b\left(y_{n}, y_{m}\right)<\rho\left(\frac{1-\gamma}{\beta+\lambda}\right)=1
$$

for each $m \in N$. Thus, we have $\left\{W_{n}\right\}$ is convergent for each $\mathrm{m} \in \mathrm{N}$, this implies $\lim _{\mathrm{n}, \mathrm{m} \rightarrow \infty} \mathrm{W}_{\mathrm{m}-1}-\mathrm{W}_{\mathrm{n}-1}=0$. Then by using Eq. 27, we get $d_{b}\left(y_{n}, y_{m}\right) \rightarrow 1$ as $n, m \rightarrow \infty$. Hence, $\left\{y_{n}\right\}$ is a Cauchy sequence. Since, $\mathrm{X}$ is complete, there exists $\mathrm{y}^{*} \in \mathrm{X}$ such that:

$$
\mathrm{d}_{\mathrm{b}}\left(\mathrm{fx}_{\mathrm{n}}, \mathrm{y}^{*}\right) \rightarrow 1 \text { and } \mathrm{d}_{\mathrm{b}}\left(\mathrm{gx}_{\mathrm{n}}, \mathrm{y}^{*}\right) \rightarrow 1 \text { asn } \rightarrow \infty
$$

Since, $\mathrm{gX}$ closed, we have $\mathrm{y}^{*} \in \mathrm{gX}$. It implies that there exists $\mathrm{x}^{*} \in \mathrm{X}$ such that $\mathrm{y}^{*}=\mathrm{gx}^{*}$. Now, we have to show that $\mathrm{x}^{*}$ is a coincidence point of $\mathrm{f}, \mathrm{g}$. Consider that from Eq. 1, we have:

$$
d_{b}\left(f x^{*}, g x^{*}\right) \leq\left(d_{b}\left(f x^{*}, f x_{n}\right)\right)^{d_{b}\left(f x^{*}, g x^{*}\right)}\left(d_{b}\left(f x_{n}, g x^{*}\right)\right)^{d_{b}\left(f x^{*}, g x^{*}\right)}
$$

By using Eq. 23, we have:

$$
\begin{aligned}
& d_{b}\left(f x^{*}, g x^{*}\right) \leq\left(d_{b}\left(f x^{*}, f x_{n}\right)\right)^{d_{b}\left(f x^{*}, g x^{*}\right)}\left(d_{b}\left(f x_{n}, g x^{*}\right)\right)^{d_{b}\left(x^{*}, g x^{*}\right)} \\
& d_{b}\left(f x^{*}, g x^{*}\right) \leq\left(\begin{array}{l}
d_{b}^{\beta}\left(f x^{*}, g x^{*}\right) \times d_{b}^{\gamma b\left(x^{*}, x_{n}\right)}\left(f x_{n}, g x_{n}\right) \times \\
d_{b}^{\lambda}\left(g x^{*}, g x_{n}\right)^{\frac{b\left(f x^{*}, g x^{*}\right)}{b\left(x^{*}, x_{n}\right)}}
\end{array}\right) \times \\
& d_{b}\left(f x_{n}, g x^{*}\right)^{d\left(f x^{*}, g x^{*}\right)}=\left(\begin{array}{l}
d_{b}^{\beta}\left(f x^{*}, g x^{*}\right) \times d_{b}^{\gamma b\left(x^{*}, x_{n}\right)}\left(y_{n}, y_{n-1}\right) \times \\
d_{b}^{\lambda}\left(y^{*}, y_{n-1}\right)^{\frac{b\left(f x^{*}, g x^{*}\right)}{b\left(x^{*}, x_{n}\right)}}
\end{array}\right) \times \\
& \left(d_{b}\left(y_{n}, y^{*}\right)\right)^{b\left(f x^{*}, g x^{*}\right)}=\left(d_{b}\left(f x^{*}, g x^{*}\right)\right)^{\beta / \frac{\left.b x^{*}, g x^{*}\right)}{b\left(y^{*}, x_{n}\right)}} \times \\
& \left(d_{b}\left(Y_{n}, Y_{n-1}\right)\right)^{\gamma b\left(x^{*}, g x^{*}\right)} \times\left(d_{b}\left(y^{*}, y_{n-1}\right)\right)^{\frac{\lambda b\left(f x^{*}, g x^{*}\right)}{b\left(x_{n}\right)}} \times \\
& \left(d_{b}\left(y_{n}, y^{*}\right)\right)^{b\left(f x^{*}, g x^{*}\right)} \leq\left(d_{b}\left(f x^{*}, g x^{*}\right)\right)^{\beta b\left(f x^{*}, g x^{*}\right)} \times \\
& \left(d_{b}\left(y_{n}, y_{n-1}\right)\right)^{\gamma b\left(f x^{*}, g x^{*}\right)} \times\left(d_{b}\left(y^{*}, y_{n-1}\right)\right)^{\lambda b\left(f x^{*}, g x^{*}\right)} \times\left(d_{b}\left(y n, y^{*}\right)\right)^{b\left(f x^{*}, g x^{*}\right)}
\end{aligned}
$$

By using Eq. 25 and 28 and for $n \rightarrow \infty$, we get $d_{b}\left(f^{*}\right.$, $\left.\mathrm{gx}^{*}\right)=1$. Thus, we have $\mathrm{fx}^{*}=\mathrm{gx}^{*}=\mathrm{y}^{*}$. Next, we have to show that $\mathrm{y}^{*}$ is a common fixed point of $\mathrm{f}$, g. Consider that from Eq. 23, we have:

$$
\begin{aligned}
& d_{b}\left(y^{*}, f y^{*}\right)=d_{b}\left(f x^{*}, f y^{*}\right) \leq\left(d_{b}^{\beta}\left(f x^{*}, g x^{*}\right)\right) \times \\
& \left.\left.d_{b}^{\gamma b\left(x^{*}, y\right)}\left(f y^{*}, g y^{*}\right) \times d_{b}{ }^{\lambda}\left(g x^{*}, g y^{*}\right)\right)\right)^{\frac{1}{b\left(x^{*}, y^{*}\right)}}=\left(d_{b}\left(y^{*}, g y^{*}\right)\right)^{\frac{\lambda}{b\left(x^{*}, y^{*}\right)}}
\end{aligned}
$$

Since, $\mathrm{f}, \mathrm{g}$ is a weakly compatible and $\mathrm{fx}^{*}=\mathrm{gx}^{*}=\mathrm{y}^{*}$ this implies:

$$
g f x^{*}=f g x^{*}=f y^{*}=g y^{*}
$$

So by using equality Eq. 29 and 30, we have:

$$
\begin{aligned}
& d_{b}\left(y^{*}, f y^{*}\right) \leq\left(d_{b}^{\beta}\left(f x^{*}, f x^{*}\right)\right) \times d_{b}{ }^{\gamma b\left(x^{*}, y\right)}\left(f^{*}, f^{*}\right) \times \\
& \left.d_{b} \lambda\left(y^{*}, f y^{*}\right)\right)^{\frac{1}{b\left(x^{*}, y^{*}\right)}}=\left(d_{b} \lambda\left(y^{*}, f y^{*}\right)\right)^{\frac{1}{b\left(x^{*}, y^{*}\right)}} \leq\left(d_{b}\left(y^{*}, f y^{*}\right)\right)^{\lambda}
\end{aligned}
$$

Thus, , we get:

$$
\left(d_{b}\left(y^{*}, f y *\right)\right)^{1-\lambda} \leq 1
$$

Since, $1-\lambda>0$, , we have $d_{b}\left(y^{*}, f y^{*}\right)=1$ that is $y^{*}=f y^{*}$. By using (29), we get:

$$
f y^{*}=g y^{*}=y^{*}
$$

Hence, $\mathrm{y}^{*}$ is a common fixed point of $\mathrm{f}$, $\mathrm{g}$. Uniqueness of common fixed point of $\mathrm{f}$, $\mathrm{g}$ can be shown as follows: suppose there exists $\mathrm{u}^{*}$ as another common fixed point of $\mathrm{f}$, g. Consider that:

$$
\begin{aligned}
& d_{b}\left(y^{*}, u^{*}\right)=d_{b}\left(f y^{*}, f u^{*}\right) \leq\left(d_{b}^{\beta}\left(f y^{*}, g y^{*}\right) d_{b}^{\gamma b\left(y^{*}, u^{*}\right) \times}\right. \\
& \left.\left(f^{*}, g^{*}\right) d_{b} \lambda\left(g y^{*}, g^{*}\right)\right)^{\frac{1}{b\left(y^{*}, u^{*}\right)}} \leq\left(d_{b}^{\beta}\left(f y^{*}, f y^{*}\right) d_{b} \gamma b\left(y^{*}, u^{*}\right) \times\right. \\
& \left.\left(f u^{*}, f^{*}\right) d_{b} \lambda\left(y^{*}, u^{*}\right)\right)^{\frac{1}{b\left(y^{*}, u^{*}\right)}}=\left(d_{b}\left(y^{*}, u^{*}\right)\right) \frac{\lambda}{b\left(y^{*}, u^{*}\right)} \leq \\
& \left(d_{b}\left(y^{*}, u^{*}\right)\right)^{\lambda}
\end{aligned}
$$

So, we get:

$$
\left(d_{b}\left(y^{*}, u^{*}\right)\right)^{1-\lambda} \leq 1
$$

Since, $1-\lambda>0$, we have $d_{b}\left(y^{*}, f y^{*}\right)=1$ that is $y^{*}=u^{*}$.

Corollary 3.1: Let $\left(X, d_{b}\right)$ be a complete extended multiplicative b-metric space $f, g: X \rightarrow X$ and be two mappings such that $\mathrm{fX} \subseteq \mathrm{gX}$, $\mathrm{gX}$ closed and satisfying the following conditions:

$$
\left(d_{b}(f x, f y)\right)^{b(x, y)} \leq d_{b}^{\lambda}(g x, g y)
$$


where $0<\lambda<1$. If $\lim _{n, m \rightarrow \infty} b\left(y_{n}, y_{m}\right)<\frac{1}{\lambda}$ and $\{f, g\}$, weakly compatible, then $\mathrm{f}$, $\mathrm{g}$ have a unique common fixed point.

Proof: By taking from Theorem 4.

Corollary 3.2: Let $\left(X, d_{b}\right)$ be a complete extended multiplicative b-metric space and $\mathrm{f}: \mathrm{X} \rightarrow \mathrm{X}$ be a mapping that satisfying the following conditions:

$$
\left(d_{b}(f x, f y)\right)^{b(x, y)} \leq d_{b}^{\beta}(f x, x) d_{b}^{\gamma b(x, y)}(f y, y) d_{b}^{\lambda}(x, y)
$$

where $\quad \beta \geq 0, \quad 0 \leq \gamma<1, \quad 0-\lambda>1, \quad 0<\beta+\lambda /(1-\gamma) \quad$ and $\lim _{n, m \rightarrow \infty} b\left(y_{n}, y_{m}\right)<\frac{1-\gamma}{\beta+\lambda}$. Then has a unique fixed point.

Proof: By taking gx $=\mathrm{x}$ from Theorem 4 .

Corollary 3.3: Let $\left(\mathrm{X}, \mathrm{d}_{\mathrm{b}}\right)$ be a complete extended multiplicative $\mathrm{b}$-metric space and $\mathrm{f}: \mathrm{X} \rightarrow \mathrm{X}$ be a mapping and satisfying the following conditions:

$$
\left(d_{b}(f x, f y)\right)^{b(x, y)} \leq d_{b}^{\lambda}(x, y)
$$

where $0<\lambda<1$ and $\lim _{\mathrm{n}, \mathrm{m} \rightarrow \infty} \mathrm{b}\left(\mathrm{y}_{\mathrm{n}}, \mathrm{y}_{\mathrm{m}}\right)<\frac{1}{\lambda}$. Then has a unique fixed point.

Proof: By taking from Theorem 3.4.

Example 3.3: As shown in Example 2.4 and by taking a $=4^{32}$ that is $\mathrm{b}(\mathrm{x}, \mathrm{y})=2+(\mathrm{x}-\mathrm{y})^{2}$ and $\mathrm{d}_{\mathrm{b}}(\mathrm{x}, \mathrm{y})=(4)^{32|\mathrm{x}-\mathrm{y}| 2}$ for all $x, y \in X=[0,1]$ and $\lambda=1 / 4, \beta=3 / 16, \gamma=1 / 16$. Let $f(x)$ $=\mathrm{x}^{4} / 2^{4}$ and $\mathrm{g}(\mathrm{x})=\mathrm{x}^{2} / 2^{2}$, for all $\mathrm{x} \in \mathrm{X}$. It is clear that $\mathrm{fX} \subseteq \mathrm{gX}$. Let $\mathrm{x} \neq \mathrm{y}$ using Theorem 3.4 consider that:

$$
\begin{aligned}
& d_{b}^{\beta}(f x, g x) d_{b}^{\lambda b(x, y)}(f y, g y) d_{b}{ }^{\lambda}(g x, g y)=d_{b}{ }^{\beta}\left(\frac{x^{4}}{2^{4}}, \frac{x^{2}}{2}\right) \times \\
& \mathrm{d}_{\mathrm{b}}^{\gamma\left(2+(\mathrm{x}-\mathrm{y})^{2}\right)} \times\left(\frac{\mathrm{y}^{4}}{2^{4}}, \frac{\mathrm{x}^{2}}{2}\right) \times \mathrm{d}_{\mathrm{b}}^{\lambda}\left(\frac{\mathrm{x}^{2}}{2}, \frac{\mathrm{y}^{2}}{2}\right)=(4)^{32 \mathrm{\beta}\left|\mathrm{x}^{4} \frac{2}{2}^{\frac{x^{2}}{2}}\right|^{2}} \times \\
& (4)^{32 \gamma\left(2+(x-y)^{2}\right)}\left|\frac{\mid y^{4}}{2^{4}} \cdot \frac{x^{2}}{2}\right|^{2} \times(4)^{32 \lambda \lambda\left|\frac{x^{2}}{2} \cdot \frac{y^{2}}{2}\right|^{2}} \geq(4)^{32 \beta \mid}\left|\frac{\mid 2^{4}}{\frac{2^{4}}{2}} \frac{x^{2}}{2}\right|^{2} \times \\
& \left.\left.(4)^{32 \gamma\left(2+(x-y)^{2}\right)}\left|\frac{y^{4}}{2^{4}} x^{2}\right|^{2}\right|^{2}=(4)^{32 \gamma\left(2+(x-y)^{2}\right.}\right)\left|\frac{y^{4}}{2^{4}}-\frac{x^{2}}{2}\right|^{2}+\frac{32}{3}(3)\left|\frac{x^{4}}{2^{4}-\frac{x^{2}}{2}}\right|^{2}
\end{aligned}
$$

Since, $x, y \in[0,1]$, we have $2+(x-y)^{2} \leq 3$. Thus by entering $\beta=3 / 16, \gamma=1 / 16$ into (31), we have:

$$
\begin{aligned}
& \left.d_{b}^{\beta}(f x, g x) d_{b}^{\gamma b(x, y)}(f y, g y) d_{b}^{\lambda}(g x, g y) \geq(4)^{2\left(2+(x-y)^{2}\right)}\right)\left(\left.\left|\frac{y^{4}}{2^{4}} x^{2}\right|^{2}\right|^{2}+\left.\left|\frac{x^{4}}{2^{4}} x^{2}\right|^{2}\right|^{2}\right) \geq \\
& \left.\left.(4)^{2\left(2+(x-y)^{2}\right.}\right)\left(\frac{1}{2}\left|\frac{x^{4}}{2^{4}} \frac{y^{4}}{2^{4}}\right|^{2}\right)=(4)^{2\left(2+(x-y)^{2}\right)}\right)\left(\left|\frac{x^{4}}{2^{4}} \frac{y^{4}}{2^{4}}\right|\right)=\left.\left.((4))^{\frac{x^{4}}{2^{4}} y^{4}}\right|^{2^{4}}\right|^{\left.\left.\right|^{\left(2+(x-y)^{2}\right.}\right)}= \\
& \left(d_{b}(f x, f y)\right)^{b(x, y)}
\end{aligned}
$$

By using $\mathrm{y}_{\mathrm{n}}=\mathrm{f}\left(\mathrm{x}_{\mathrm{n}}\right)=\mathrm{g}\left(\mathrm{x}_{\mathrm{n}+1}\right), \mathrm{f}(\mathrm{x})=\mathrm{x}^{4} / 2^{4}$ and $\mathrm{g}(\mathrm{x})=$ $x^{2} / 2^{2}$, we have $y_{0}=f\left(x_{0}\right)=x^{4} / 2^{4}=g\left(x_{1}\right)$ thus, $x_{1}=x^{2} / 2^{2}, y_{1}$ $=\mathrm{f}\left(\mathrm{x}_{1}\right)=\mathrm{f}\left(\mathrm{x}^{2} / 2^{2}\right)=\mathrm{x}^{8} / 2^{8}=\mathrm{g}\left(\mathrm{x}_{2}\right)$ thus, $\mathrm{x}_{2}=\mathrm{x}^{4} / 2^{3}, \mathrm{y}_{2}=\mathrm{f}\left(\mathrm{x}_{2}\right)$ $=f\left(x^{4} / 2^{3}\right)=x^{16} / 2^{16}=g\left(x_{2}\right)$ thus, $x_{3}=x^{8} / 2^{7}$. Generally, , we have:

$$
y_{n}=\frac{x^{2^{n+2}}}{2^{n+2}}, x_{n}=\frac{x^{2^{n}}}{2^{2^{n}-1}}
$$

By using $b(x, y)=2+(x-y)^{2}$ and since, $x \in[0,1]$, we have:

$$
\begin{aligned}
& \lim _{n, m \rightarrow \infty} b\left(y_{n}, y_{m}\right)=\lim _{n, m \rightarrow \infty} 2+y_{n}+y_{m}=\lim _{n, m \rightarrow \infty} 2+\frac{x^{2^{n+2}}}{2^{n+2}}+ \\
& \frac{x^{2^{m+2}}}{2^{m+2}}=2<\frac{1-\gamma}{\beta+\lambda}=\frac{15}{7}
\end{aligned}
$$

Since, $x \in[0,1]$, the sequence $\left\{y_{n}\right\} \frac{x^{2^{n+2}}}{2^{n+2}}$ is convergent to 0. Indeed:

$$
\lim _{n, m \rightarrow \infty} d_{b}\left(y_{n}, 0\right)=\lim _{n, m \rightarrow \infty}\left(\frac{x^{2^{n+2}}}{2^{n+2}}, 0\right)=\lim _{n, m \rightarrow \infty}(4)^{32}\left|\frac{x^{2^{n+2}}}{2^{n+2}}\right|^{2}=1
$$

Thus, based on Theorem 3.4, , we conclude that $\mathrm{x}^{*}=$ 0 is a unique common fixed point of $\mathrm{f}$, $\mathrm{g}$.

\section{CONCLUSION}

In this study, we establish that there exists sufficient condition for existence and uniqueness of fixed point on generalized multiplicative contraction mappings and common fixed point for two functions on generalized multiplicative contraction in extended multiplicative b-metric spaces.

\section{REFERENCES}

Abbas, M., B. Ali and Y.I. Suleiman, 2015. Common fixed points of locally contractive mappings in multiplicative metric spaces with application. Int. J. Math. Math. Sci., Vol. 2015, $10.1155 / 2015 / 218683$

Abdou, A.A.N., 2016. Fixed point theorems for generalized contraction mappings in multiplicative metric spaces. J. Nonlinear Sci. Appl., 9: 2347-2363.

Ali, M.U., T. Kamran and A. Kurdi, 2017. Fixed point theorems in b-multiplicative metric spaces. U.P.B. Sci. Bull. Ser A. Applied Math. Phys., 79: 107-116.

Bashirov, A.E., E.M. Kurpinar and A. Ozyapici, 2008. Multiplicative calculus and its applications. J. Math. Anal. Appl., 337: 36-48. 
Gu, F. and Y.J. Cho, 2015. Common fixed point results for four maps satisfying $\varphi$-contractive condition in multiplicative metric spaces. Fixed Point Theory Appl., Vol. 2015, No. 1. 10.1186/s13663-015-0412-4

He, X., M. Song and D. Chen, 2014. Common fixed points for, weak commutative mappings on a multiplicative metric space. Fixed Point Theory and Appl., Vol. 2014, No. 1. 10.1186/1687-1812-2014-48

Khan, A., H. Khan, D. Baleanu, H. Jafari, T.S. Khan and M. Alqurashi, 2017. A fixed point theorem on multiplicative metric space with integral-type inequality. J. Math. Comput. Sci., 18: 18-28.
Khan, Q.H. and M. Imdad, 2016. Coincidence and common fixed point theorems in a multiplicative metric space. Adv. Fixed Point Theory, 6: 1-9.

Kumar, P., S. Kumar, S.M. Kang and C.Y. Jung, 2016. Common random fixed point theorems in multiplicative metric spaces. Int. J. Math. Anal., 10: 399-413.

Singh, S.R., M. Ughade, R.D. Daheriya, R. Jain and S. Shrivastava, 2016. Coincidence points \& common fixed points for multiplicative expansive type mappings. J. Adv. Math. Comput. Sci., 19: 1-14. 